\title{
Impediments to implementing evidence-based mental health in developing countries
}

M ental and behavioural disorders are among the most important causes of morbidity and disability in both the developed and the developing world. ${ }^{1}$ As a proportion of total morbidity and disability, these problems are comparatively less significant in developing countries, mainly because of the large burden of communicable, maternal, perinatal, and nutritional conditions in these regions. However, mental disorders result in major deprivations such as lower levels of educational attainment, unemployment and, in extreme cases, homelessness of patients. ${ }^{1}$

The delivery of healthcare services including mental health in low and middle income countries presents a number of difficult challenges such as population explosion, unplanned urbanisation, scarcity of human resources, lack of reliable data, and lack of a systematic approach to healthcare distribution and referral systems. ${ }^{2}$ Clinicians attempting to practice evidence-based mental health face additional challenges. In the model proposed for evidence-based decision making, ${ }^{3}$ consideration of the clinical and physical circumstances are synthesised with research evidence and patients' preferences, while clinical expertise is required to assemble these additional elements to provide optimal treatment. Given the different components of this model, we believe that existing differences could hinder application of evidence based practice in the developing world. In this report, we discuss some of these factors, emphasising those that can be changed.

\section{SOME FUNDAMENTAL OBSTACLES}

Prevention in mental health care is intimately linked with overall human and community development. The low priority and the limited resources for provision of mental health care raise two noticeable problems in developing countries. ${ }^{4}$ Policy makers generally place more emphasis on mortality oriented statistics, which are rather low for mental disorders. Recent studies, however, have tried to change this attitude with warnings that mental illnesses constitute up to $12 \%$ of the global burden of disease ${ }^{5}$ and that there are consistent relations between these disorders and widely prevalent factors such as low educational levels, financial insecurity, poor physical health, and rapid social change. ${ }^{\circ}$

The key to combating the growing divergence between needs and mental health services in developing countries is the reinforcement of primary care facilities. ${ }^{7}$ In our country, Iran, a national mental health programme has been formulated for universal coverage of services and integration of mental health care within primary care; however, the effectiveness of this programme is disputed and needs further evaluation. ${ }^{8}$

The small number of people in developing countries who do research has also adversely affected the quality of medical research and education. ${ }^{9}$ Less than $10 \%$ of the current global funding is allocated for research on disorders that affect over $90 \%$ of the world population (the 10/90 gap). ${ }^{10}$ This has also diverted researchers in developing countries towards the priorities and interests of industrialised countries. ${ }^{11}$ The ratio of medical students to teachers does not permit small group sessions and problem based learning. ${ }^{12}$ Neuropsychiatric skills are taught in tertiary care clinics using patients referred for specialist interventions. ${ }^{13}$ New medical graduates, however, have to work in overcrowded primary care clinics and their acquired skills may not help them in real outpatient practice, leading to a wide gap between what they are taught and what they need to know.

Pharmaceutical industries here have more power to influence the outlook of uneducated people and impress practitioners who do not have the skills to critically appraise the evidence. ${ }^{9}$ Continuing medical education programmes that are conducted mainly with the support (and control) of pharmaceutical companies may also mislead physicians when choosing the best treatment. ${ }^{14}$ The situation is aggravated by the lack of effective policy for marketing approval of drugs. Drugstores are often flooded with a myriad of products, listed for questionable indications. ${ }^{14}$

\section{SOCIAL AND CLINICAL CIRCUMSTANCES}

Developing countries lack well organised government funded healthcare referral systems. ${ }^{15}$ The first line consultant to a mentally ill patient in a developing country could be: a traditional or religious healer, an alternative medic, a general practitioner, or even a specialist or subspecialist. ${ }^{16}$ Currently, in many parts of the developing world, people believe in traditional healers rather than experienced healthcare practitioners. ${ }^{17}$ The popularity and public acceptability of alternative and herbal medicine in the developing world has led many physicians to use these therapies to attract patients.

Busy clinics, either primary or secondary, put immense pressure on both physicians and patients. Sound doctorpatient relationships become almost impossible in this scenario. Making accurate diagnoses and proper follow up in the view of large number of patients, time constraints, and wide range of diseases are almost impracticable for novices (considering their background knowledge and skills). This leads to a high rate of clinical errors and superfluous diagnostic tests.

\section{DIFFICULTIES IN RESEARCH-BASED CLINICAL PRACTICE \\ Sources of evidence}

In most areas of the developing world, the major obstacle to evidence-based practice is that physicians have limited access to, or familiarity with, computers, the Internet, searchable databases, and library facilities for full text retrieval of journal articles and guidelines. ${ }^{18}$ The current problem with evidence-based medicine in developed countries may be the

Abbreviations: LCGs, local clinical guidelines; RCTs, randomised controlled trials. 
lack of randomised clinical trials (RCTs) in many areas, but here the major problem is ignorance and unawareness of physicians of the many existing RCTs and systematic reviews. ${ }^{19}$ These limitations ultimately force the practice of medicine using lower levels of evidence such as experts' opinions and outdated textbooks. Along with dubious validity, these sources of information could also delay incorporation of new concepts in various areas of medicine. ${ }^{20}$

\section{Skills to appraise the evidence}

Evidence-based medicine is still an indefinite term for a large body of practicing physicians in developing countries. In our country, we have noticed that a major problem influencing many aspects of science, including medicine, is the lack of a "culture of appraising and being appraised", especially among those who have been named "experts". Many practitioners are unfamiliar with the basic concept of uncertainty in medical decisions. The very few users of evidence based medicine, considered as a relatively new product of the old basic science (clinical epidemiology), ${ }^{21}$ are mainly some enthusiastic academic researchers. In our country, the view of many clinicians is that epidemiology is something about the incidence and prevalence of infectious diseases (attributed to the word epidemic) and therefore not considered worthy of application in clinical practice. As even the principles of this science have not yet been integrated in the usual medical curricula of students in developing countries, many clinicians would reject learning skills of critical appraisal in the postgraduate medical courses. ${ }^{18}$

\section{Applying the evidence}

Conducting research to gain patient oriented outcomes is a costly and time consuming venture. Organising these studies also needs competent systems, equipped settings, trained personnel, patients with some levels of literacy, and, most importantly, funding. In developing countries, however, despite attempts at patient oriented research and limited systematic reviews the main body of evidence comes from developed countries, which should be evaluated with caution for applicability to people in developing countries.

Several factors limit the cross cultural applicability of research findings in psychiatry. ${ }^{22}$ Pathophysiological mechanisms for many mental illnesses are yet to be defined and these diseases could be different naturally from their occidental twins in various ways such as presentation, terminology, and course. In Iran, for instance, patients mostly use somatic complaints to verbalise their psychological problems, and metaphors and figurative language are frequently used in this regard. ${ }^{23}$ Many diagnostic categories for common mental disorders, usually developed in Western countries, may have limited validity at this point.

\section{PATIENTS' PREFERENCES AND CHARACTERISTICS}

Public opinion about psychiatric patients is usually very negative in the developing world. In many cultures, patients with mental illnesses are considered "dangerous" and "incapable of performing any useful social role". Mental disorders are looked upon as "untreatable" originating from "metaphysical sources". ${ }^{24}$ As a result, case records requiring health workers to state the diagnosis show far lower numbers of mental disorders than expected from epidemiological studies. $^{25}$ This may lead logically to decreased physician involvement and demands on care, at least in the early stages of disease. Consequently, many patients present in the primary care setting with various complications due to lack of earlier treatments. ${ }^{26}$
The characteristics of patients in developing countries (such as family and social support) may alter response to a specific treatment. In low income countries with insurance systems where the bulk of healthcare expenditures continue to be shouldered by individual patients or their families, patients' attitudes and compliance to therapies differ grossly from those of developed countries. Cultural norms and beliefs also play their role here. In a study on 150 randomly selected psychiatric outpatients in Iran, for instance, $37.4 \%$ had history of early discontinuation of psychotropic medications, mainly because of the prevalent belief that these drugs are addictive. ${ }^{27}$

\section{SOME SUGGESTIONS}

Limitations of developing countries in various directions should be observed rationally. In a country where practitioners need to provide health care at less than $£ 7$ (\$13) per person year, ${ }^{14}$ strategies employed in industrialised nations should not be expected to succeed. To overcome some fundamental problems such as the nature and rising prevalence of mental diseases, public opinion of these patients, and medical training and research shortfalls, some changes are required in basic aspects of the healthcare system and culture of these countries, which seem unattainable at least in the short term. Focusing on achievable goals can be a logical first step.

Cultivation of a culture of "appraising and being appraised" should begin at basic levels of the community such as in schools. Though it may appear to be far fetched, our ultimate ambition is to enter some essentials of problem solving and decision making into the students' syllabus. Moreover, in developing countries, mass media (especially television) could play a great role in constructively changing public attitudes and behaviour. The approach to epidemiology in undergraduate medical curricula should be modified and fundamentals of critical appraisal and information mastery should be inserted. Governments also need to reform health departments, particularly to properly manage the healthcare market, to control the quality of generic drugs, and to include mental health perceptions in primary care settings. In our country, approving and importing drugs are under control of a group of experts who may not know much about principles of cost effective analysis.

Given the problem of limited time and high workload of physicians, application of pre-appraised journals with brief commentaries and local clinical guidelines (LCGs) should be encouraged. Particularly for university hospitals, we suggest that some academics be employed to generate these LCGs, using their original data and best available external evidence. In each academic ward, one or two "clinical scientists"28 could work to generate LCGs and other (evidence-based) clinicians can use their products. The private practitioners should then approach the drug representatives' evidence and advertising brochures more cautiously.

In our experience (which may also be biased!), physicians can be divided into two major categories concerning their reaction when introduced to evidence-based medicine. The first group typically rejects the concept and guards against it. Their claim is, if evidence-based practice is right, my practice thus far would be completely erroneous. The other group, however, accepts the concept and feels impelled to investigate and apply it thoroughly. In our opinion, introducing evidence-based medicine with statistical points of refining probability or various sophisticated checklists of critical appraisal would lead more practitioners to reject evidencebased practice. These details should be postponed for later 
66

stages, especially for fervent practitioners. Considering time limits and the unavailability of many full text articles in developing countries, the practicality of using very concise checklists and teaching skills to critically appraise medical abstracts is of concern.

Experts' opinions, textbooks, and traditional review articles are the most acknowledged (and sometimes idolised) sources of evidence in developing countries, and warning about numerous possible biases in these sources is especially vital. As these are the most commonly used sources of information, programmes intending to teach practical skills of critical appraisal should focus mainly and firstly on these areas. Teaching assessment of expert-based sources such as textbooks, seminars, ward rounds, and drug brochures should be prioritised in the workshops. Physicians have to be instructed to search for higher levels of evidence, at least, when encountering inconsistencies in their customary sources.

Pending all that is mentioned above, to begin with, enthusiastic groups from either the private or public sector could popularise the concept of evidence-based medicine possibly via workshops for medical professionals, effective use of mass media, and by their efficient application of the best available evidence, even from its lowest levels.

\section{ACKNOWLEDGEMENTS}

The authors acknowledge Dr Akhoondzadeh, Dr Larijani, and Dr Sharifi from Psychiatry Research Center of Tehran University of Medical Sciences for their valuable comments on the previous drafts of this article.

A SOLTANI, A MOAYYERI, M RAZA Endocrinology \& Metabolism Research Center, Shariati Hospital, Tehran, Iran

Correspondence to: Alireza Moayyeri, Endocrinology and Metabolism Research Center, Shariati Hospital, Northern Kargar Ave, Tehran 14114, Iran; moayyeri@doctor.com

All authors are members of the Evidence Based Medicine Working Team.

\section{REFERENCES}

1 World Health Organization. World health report 2001. Geneva: WHO, 2001.

2 Desjarlais R, Eisenberg L, Good B, et al. World mental health. Problems and priorities in low-income countries. Oxford: Oxford University Press, 1995.
3 Haynes RB, Devereaux PJ, Guyatt GH. Clinical expertise in the era of evidence-based medicine and patient choice. ACP J Club 2002;136:A1 1-14. 4 Thornicroft G, Maingay S. The global response to mental illness. BMJ 2002;325:608-9.

5 Murray CJ, Lopez AD. Global mortality, disability, and the contribution of risk factors: Global Burden of Disease Study. Lancet 1997;349:1436-42.

6 Patel V, Kleinman A. Poverty and common mental disorders in developing countries. Bull World Health Organ 2003:81:609-15.

7 World Health Organization. The introduction of a mental health component into primary health care. Geneva: WHO, 1990.

8 Yasamy MT, Shahmohammadi D, Bagheri Yazdi SA, et al. Mental health in the Islamic Republic of Iran: achievements and areas of need. East Mediterr Health J 2001:7:381-91.

9 Bhutta Z. Practicing just medicine in an unjust world. BMJ 2003;327:1000-1.

$10 \mathrm{Ad} \mathrm{Hoc} \mathrm{Committee} \mathrm{on} \mathrm{Health} \mathrm{Research.} \mathrm{Investing} \mathrm{in} \mathrm{health} \mathrm{research} \mathrm{and}$ development. Geneva: World Health Organization, 1996.

11 Wolffers I, Adjei S, van der Drift R. Health research in the tropics. Lancet 1998:351:1652-4

12 Ardalan A, Shahmohammadi D. Psyciatrist manpower in Iran: a planning evidence. Andeesheh Va Raftar 2002;8:13-14 (in Persian)

13 Jacob KS. Community care for people with mental disorders in developing countries: problems and possible solutions. Br J Psychiatry 2001;178:296-8.

14 Paul Garner, Rajendra Kale, Rumona Dickson, et al. Getting research findings into practice: Implementing research findings in developing countries. BMJ 1998;317:531-5

15 World Health Organization. Hospitals and health for all. Technical Report Series No. 744. Geneva: WHO, 1987.

16 Kleinman A. Patients and healers in the context of culture. An exploration of the borderland between anthropology, medicine and psychiatry. Berkeley, CA: University of California Press, 1980.

17 Patel V, Abas M, Broadhead J, et al. Depression in developing countries: lessons from Zimbabwe. BMJ 2001;322:482-4.

18 Dans AL, Dans LF. The need and means for evidence-based medicine in developing countries. Evid Based Med 2000;5:100-1.

19 Bleck TP. Alternatives to evidence based medicine. Different rating scale could be used. BMJ 2000;321:239.

20 Antman EM, Lau J, Kupelnick B, et al. A comparison of results of metaanalyses of randomized control trials and recommendations of clinical experts. Treatments for myocardial infarction. JAMA 1992;268:240-8.

21 Sackett DL. Clinical epidemiology: what, who, and whither. J Clin Epidemiol 2002:55:1161-6.

22 Thornicroft G, Sartorius N. The course and outcome of depression in different cultures: 10-year follow-up of the WHO Collaborative Study on the Assessment of Depressive Disorders. Psychol Med 1993;23:1023-32.

23 Davidian H, Shahmohammadi D, Azordeghan F, et al. Common symptoms and signs of psychiatric disorders in Iranian culture. Hakim Res $J$ 2001;4:11-12 (in Persian).

24 Mohit A. Mental health and psychiatry in the Middle East: historical development. East Mediterr Health J 2001;7:336-47.

25 Patel V. Recognizing common mental disorders in primary care in African countries: should "mental" be dropped? Lancet 1996;347:742-4.

26 Ehsanmanesh M. Epidemiology of mental disorders in Iran: a review of research findings. Anddesheh Va Raftar 2001;6:54-69 (in Persian).

27 Nejad AG, Pouya F. Medication noncompliance among psychiatric outpatients in Iran. Can J Psychiatry 2003:48:571.

28 Slawson DC, Shaughnessy AF. Becoming an information master: using POEMs to change practice with confidence. J Fam Pract 2000;49:276. 